\title{
Spontaneous Transfer of Matching by Infant Chimpanzees (Pan troglodytes)
}

\author{
David L. Oden \\ La Salle University
}

\author{
Roger K. R. Thompson \\ Franklin and Marshall College
}

\author{
David Premack \\ University of Pennsylvania
}

\begin{abstract}
Four infant chimpanzees learned a matching-to-sample task when only two training stimuli were used. They then spontaneously transferred the matching concept to novel items, including threedimensional objects and fabric swatches, without any experimenter-provided differential feedback. These results support the view that the matching concept is broadly construed by chimpanzees from the beginning and does not depend upon explicit training.
\end{abstract}

Humans continually make judgments of similarity and identity across a broad range of domains and at all levels of abstraction (Wallach, 1958). From early childhood, we spontaneously perceive identity relations between objects (Daehler, Lonardo, \& Bukatko, 1979; Ricciutti, 1965) and events (Johansson, 1964) and across modalities (Meltzoff \& Borton, 1979; Spelke, 1979). We detect similarities and differences both in time and space, and we detect identical analogical and functional relations (e.g., Shallice, 1972; Sternberg, 1977). Judgments of psychological similarity and identity are inherent also in our attributions of states of mind, attitudes, and intentions (Premack \& Woodruff, 1978), as well as in the use of linguistic metaphor (Lashchyk, 1986).

Given the pervasiveness of these issues in human behavior, it is not surprising that questions of whether and how other organisms perceive and use identity relations are among the more ubiquitous issues in comparative cognition today (D'Amato, Salmon, \& Colombo, 1985; D'Amato, Salmon, Loukas, \& Tomie, 1986; Premack, 1978, 1983a). The functional significance of detecting and acting upon constancy and change across time and space presumably is self-evident. The degree to which different species are sensitive to same/ different relations, both concrete and of varying abstraction, is less obvious. Also of interest are the conditions necessary for the expression of these abilities.

Our understanding of how other species perceive and use identity relations comes largely from studies of matching-tosample (MTS) performances. In the standard MTS task, the subject first observes a sample stimulus and is then rewarded

This research was supported by Grant BNS 8418942 from the National Science Foundation (NSF) to David Premack. Preparation of this article was supported by a grant to David L. Oden and Roger K. R. Thompson from the NSF program for support of small college faculty through association with faculty at large institutions.

We thank P. Durlach, B. Dennis, S. Sharp, P. Spritzer, and J. Altman for their help in collecting data.

Correspondence concerning this article should be addressed to David L. Oden, Department of Psychology, La Salle University, Philadelphia, Pennsylvania 19141. for responding to a choice or comparison stimulus identical to (i.e., matching) the sample. After acquisition, the generality of the matching concept is inferred from the subject's ability to match novel items in transfer tests.

Thus far, comparative studies have focused largely on the MTS ability of birds and nonhuman primates. Pigeons readily learn to match visual stimuli, but investigators differ in their interpretation of results from transfer tests (cf. Carter \& Werner, 1978; D'Amato et al., 1986; Edwards, Jagielo, \& Zentall, 1983; Premack, 1983b; Zentall \& Hogan, 1978). Zentall and his associates, for example, have argued that their subjects' ability to learn subsequent matching or oddity problems faster than their first is evidence of a generalized matching concept (Zentall, Edwards, Moore, \& Hogan, 1981; Zentall \& Hogan, 1974; Zentall \& Hogan, 1978).

Results from other studies suggest that MTS performances by pigeons largely reflect their acquiring an approach/avoid rule dependent more upon absolute stimulus values than on physical similarity (e.g., Farthing \& Opuda, 1974; Holmes 1979; Wilson, Mackintosh, \& Boakes, 1985a). D'Amato et al. (1985) concluded that responding by pigeons during transfer tests often could be interpreted in terms of such factors as stimulus generalization and common absolute coding of training and transfer stimuli. Thus far, the most convincing evidence for generalized MTS in birds comes from a study in which a corvid species, the European jay, showed clear evidence of transfer to line orientation matching or oddity after training on matching or oddity to color (Wilson, Mackintosh, \& Boakes, 1985b). Pigeon subjects in the same study showed no evidence of transfer.

In contrast to pigeons, both old- and new-world monkeys (e.g., D'Amato \& Salmon, 1984) generalize their visual MTS performances to novel stimuli readily. These results indicate that monkeys attend to physical similarity of visual stimuli and are influenced less by other factors, like absolute stimulus values, when learning to match. Comparable results have been reported for the bottlenose dolphin (Tursiops truncatus) in an auditory MTS task (Herman \& Gordon, 1974). This generalized MTS ability is greatly facilitated by several training procedures including the use of a large stimulus set, if not 
trial-unique objects, during acquisition training and continued partial differential reinforcement during transfer tests (Mishkin \& Delacour, 1975; Overman \& Doty, 1980; Worsham, 1975). If one ventures outside the visual domain to, say, audition or touch, a monkey's ability to demonstrate generalized MTS is fragile at best (D'Amato \& Colombo, 1985; Milner, 1973; Steele \& Bauer, 1983; Thompson, 1980).

The ability of chimpanzees to generalize their matching performances is even more robust than that demonstrated for monkeys. For example, Nissen, Blum, and Blum (1948) reported that all 4 of their chimpanzee subjects immediately matched new items after acquisition of the task with only two training stimuli. Such a result is obtained rarely with monkeys (cf. D'Amato, et al., 1985; Fujita, 1982, 1983; Jackson \& Pegram, 1970a, 1970b; Mello, 1971). For example, D'Amato et al. (1985) reported that after his 8 animals learned to match two training stimuli, only 2 monkeys satisfied a performance criterion of at least $70.8 \%$ correct responses in the first 24 trial transfer test. Two additional subjects subsequently met this criterion in the second, third, and fourth sessions. The chimpanzees' ability to generalize matching after training with only two items is reminiscent of studies with children (Weinstein, 1941). This ability is impressive because it implies that the chimpanzee, like the child, spontaneously uses physical similarity as the perceptual basis for matching in the absence of explicit instruction. Despite the explicit saliency of absolute stimulus values (e.g., see red, match red) the chimpanzee nevertheless adopts a more widely applicable matching rule.

Demonstration of a generalized matching concept by an animal raises two theoretical issues. One is the question of how the concept is represented, if at all. For example, an animal using physical resemblance for matching could represent it by using an abstract code of same/different. Alternatively, its choice of the correct matching stimulus may be based on having seen the sample stimulus before (e.g., old vs. new; familiar vs. unfamiliar [Premack, 1978, 1983a; 1983b]).

The second issue, regardless of the nature of the representational code, concerns the conditions necessary for acquisition of the widely applicable matching concept. One possibility is that the matching concept is broadly construed from the beginning. That is, after acquisition, the animal spontaneously applies it to new objects, categories, and/or domains. This interpretation, suggested by Premack (1983a, 1983b), is to be contrasted with the view that animals initially learn a narrowly construed matching concept during MTS training and must be taught to broaden its application to new items and categories (e.g., Fodor, 1975). The present study focuses on this latter issue of breadth of construal, and not on the nature of the code.

At first glance, Nissen et al.'s (1948) results seem to provide strong support for Premack's view that the matching concept is broadly construed from the beginning. However, this conclusion is perhaps premature because of the transfer procedures they used. In that study the subjects were differentially rewarded for correct MTS responses during the transfer tests. This procedure leaves open the possibility that the animals' performances reflected acquisition of a learning set in which an initially narrowly construed matching concept was applied to an increasingly broader class of objects. The same criticism can be directed at other studies demonstrating generalized MTS performances in both chimpanzees and monkeys (Finch, 1942; Riesen \& Nissen, 1942). Fodor's position that the matching concept is narrowly construed and broadens with experience is supported also by the finding that training with large stimulus sets facilitates MTS transfer to novel items on Trial 1 by both monkeys and a dolphin (Herman \& Gordon, 1974; Mishkin \& Delacour, 1975).

Here we report a stronger demonstration that the matching concept is broadly construed from the beginning by chimpanzees. In this study we trained naive infant chimpanzees to match by using differential reward with only two objects. Subsequently we looked at their ability to transfer spontaneously the matching concept to novel items in the absence of differential feedback from the experimenter. During the transfer tests, we rewarded the chimpanzees regardless of the correctness of their responses. We hypothesized that if their matching concept was broadly construed to begin with, as suggested by Premack (1983a, 1983b), then their performances should not suffer when tested with new objects despite the absence of experimenter-provided differential feedback.

\section{Method}

\section{Subjects}

The subjects were 4 captive-born infant chimpanzees (Pan troglodytes), 3 female (Frieda, Liza, \& Opal) and 1 male (Whiskey), between 14 to 16 months of age at the time training began. They were naive with respect to MTS procedures. Their previous experimental history consisted of testing on habituation procedures involving their viewing or handling objects singly and in pairs (Oden, Thompson, \& Premack, 1988).

The animals' daily routine consisted of morning and afternoon test sessions interspersed with free-play periods and nap time. A variety of standard infants', toddlers', and children's toys were available to the chimpanzees during their play periods. Our extended observations of the chimpanzees with their toys failed to reveal any systematic use of these objects by any animal. Typically, in the presence of their caretakers the chimpanzees ignored their toys and initiated roughand-tumble play, or they solicited care-giving, such as mutual grooming.

Between tests they were housed together in a playroom $(2.74 \mathrm{~m} \times$ $3.35 \mathrm{~m}$ ) or, weather permitting, in an outdoor $1 / 2$-acre $(0.2$ hectare) compound. At night they were housed in pairs in stainless steel cages $\left(71 \mathrm{~cm}^{3}\right)$. They were given a breakfast of pureed monkey chow and evaporated milk, and a dinner consisting of monkey chow, fresh fruits, and vegetables.

\section{Apparatus}

During the sessions of both acquisition training and transfer testing, the subjects were tested in pairs. Each animal was placed on one side of a modified baby crib $(130 \mathrm{~cm} \times 71 \mathrm{~cm} \times 56 \mathrm{~cm})$ divided by a clear Plexiglas partition, which afforded the maximum degree of separation the chimpanzees could tolerate without considerable emotional distress. The front of the crib consisted of $2.5-\mathrm{cm}$ vertical metal bars, spaced about $9 \mathrm{~cm}$ apart; the rear wall was clear Plexiglas. The crib floor was masonite, and a removable metal grid made up the ceiling. A $10-\mathrm{cm}$ gap between the crib floor and front bars allowed both subject and trainer ready access to objects in each other's work 
areas. Each half of the crib contained a blanket. The crib stood in a windowless $(2.13 \mathrm{~m} \times 3.35 \mathrm{~m})$ cinderblock room.

A gray enameled baking dish $(20 \mathrm{~cm} \times 20 \mathrm{~cm} \times 5 \mathrm{~cm})$ was mounted in the center of a small $(20 \mathrm{~cm} \times 60 \mathrm{~cm})$ table so that it could be positioned just outside the crib, level with the crib floor. The objects used during acquisition training consisted of identical stainless steel $1 / 4$-cup $(60 \mathrm{ml})$ measuring cups and identical brassplated sliding bolt locks $7.6 \mathrm{~cm}$ in length. In two of the subsequent transfer tests, assorted toys and small hardware items, (e.g., rubber hose, plastic toy dish, wooden block) were used as test objects. Fabric swatches $\left(15 \mathrm{~cm}^{2}\right)$, varying in color and texture, were used in two fabric transfer tests, and bite-sized pieces of low-preference foods (e.g., monkey chow, potato, carrot) were used in the food transfer tests.

\section{Procedure}

Object familiarization. The subjects were first familiarized with training objects and the response requirements in a free-play situation. All 4 subjects were allowed to roam freely in a playroom $(2.13 \mathrm{~m} \times$ $3.35 \mathrm{~m}$ ) with one or two trainers. Six locks and six cups were available along with two baking tins. Subjects were encouraged to handle the locks and cups, and the response of placing them into an empty baking tin was modeled. When a chimpanzee put an object into a baking tin, he or she was verbally praised and hugged by a trainer. Within six 30-min sessions, all 4 chimps learned to place objects into baking tins when encouraged to do so.

Acquisition training. Training on the MTS task in the "crib-room" began as soon as all chimpanzees placed objects into baking tins when encouraged to do so by a trainer. Each trial commenced when the trainer handed the sample object for that trial to the subject. Immediately after the chimpanzee placed the sample into the baking tin,the trainer placed the two alternative objects inside the crib, $20 \mathrm{~cm}$ apart and equidistant from the pan. If the subject placed the correct matching alternative into the pan with the sample, she or he was rewarded with effusive praise, tiekling and cuddling, and depending upon the animal's preference, a mouthful of fruit, yogurt, or fruit juice. If the subject incorrectly placed the nonmatching alternative into the pan with the sample, the trainer removed the incorrect object from the pan and replaced it with the correct matching object.

Each session consisted of 12-24 trials, depending on the trainer's judgment of the subject's level of interest and emotionality. The intertrial interval following both correct and incorrect responses averaged $30 \mathrm{~s}$, ranging from 12 to $40 \mathrm{~s}$, depending on the facility with which the experimenter recorded the data and set up the next trial Sessions were run one to three times daily, 5 days a week. Within a session, both objects-lock and cup-appeared as sample an equal number of times in a random order. Also, each alternative appeared quasi-randomly to the left and right of the subject with equal frequency.

When a subject met the predetermined criterion level of $10 / 12$ or more correct responses $(p<.05$, binomial test) it was given a series of transfer-test sessions.

Transfer tests. Each transfer test consisted of a 12-trial session in which two novel objects were both used equally often as correct and incorrect alternatives. A subject was rewarded with praise and food on every trial regardless of the correctness of a matching response. Previous experience with other chimpanzee subjects revealed that in the absence of any rewards, as in an extinction procedure, they rapidly ceased task-related responding and threw a tantrum or sulked. With the exception of this nondifferential reward procedure, the sequence of events within trials was the same as during acquisition training. The animals were tested by trainers who were familiar and unfamiliar and who were correspondingly experienced or naive with respect to the experimental program.
A total of six transfer tests was given to each subject. There were two sessions each of objects, fabrics, and food. Thus, a total of 12 novel items (e.g., 4 new objects, 4 fabrics, and 4 food items) were used altogether for each chimpanzee in the transfer-test series. They had never encountered these test objects in the test context. Each subject encountered a unique set of novel items selected from a large stimulus set so as to equate preferences for the two alternatives in a given session.

Preferences were determined a priori for each subject in a free-play or home-cage context. We determined these preferences by (a) measuring the amount of time spent interacting with an object and (b) recording the animal's initial choice in an unrewarded paired comparison forced-choice task. Care was taken to ensure that the dimensions on which the transfer items differed were orthogonal to the differences between the original two training stimuli. Preexposing the animals to the transfer stimuli also controlled for potential initial disruption of MTS performances induced by stimulus novelty (e.g., Jackson \& Pegram, 1970a).

Control for experimenter cuing. To rule out the possibility that unintentional cues from the experimenter signaled the correct response to the chimpanzees during transfer testing, we tested each subject on a more conceptual relational MTS task that nonlanguagetrained chimpanzees fail to perform successfully (Premack, 1983a). If success on the transfer MTS tests in the present study was attributable to experimenter cuing, independently of the stimulus objects, then the chimpanzees might reasonably have been expected to succeed on this more conceptually abstract task. As in the transfer tests, familiar, experienced, and unfamiliar, naive trainers were used in this phase of the study.

Retaining the same procedures used with the transfer tests (i.e., nondifferential feedback), we changed only the stimuli-pairs of objects mounted on masonite-for 200 trials with each subject. In this procedure a pair of identical objects (e.g., AA) served as the sample. The two comparison stimuli consisted of a pair of dissimilar objects (e.g., CD) and a pair of identical objects (e.g., BB), all of which differed physically from the sample. Fifty additional trials were run on the relational MTS task in which the experimenter explicitly provided cues-including pointing, looking, and body orientationas to the correct response.

\section{Results}

The number of trials to criterion with the lock and cup training items for each subject is shown in Table 1, together with their respective correct MTS performance levels for the final 24 trials preceding the first transfer test. The mean number of trials to the criterion of $p<.05$, binomial test, was 816; the mean percentage of correct responses for the final 24 trials was $86.5 \%$. Each animal's correct MTS performance level for the subsequent tranfer tests is shown also in Table 1. The mean percentages of correct MTS responses for the object, fabric, and food transfer sessions were $85.4 \%, 84.4 \%$, and $67.7 \%$ respectively.

A repeated measures analysis of variance, $F(3,9)=4.67$, $p<.05$, and subsequent Newman-Keuls tests $(p<.05)$ on ordered means showed, as suggested by Table 1, that performance levels in the transfer tests with novel objects and fabrics did not differ significantly from the $86.5 \%$ performance level obtained on the final 24 training trials. However, correct matching performance on the transfer sessions with food items differed significantly from those obtained in the transfer conditions with objects or fabrics and the final 24 training trials. 
Table 1

Trials to Criterion and Matching-to-Sample (MTS) Accuracy During Training Sessions and Transfer Sessions

\begin{tabular}{cccc}
\hline & \multicolumn{3}{c}{ MTS accuracy } \\
\cline { 2 - 4 } $\begin{array}{c}\text { Subject \& test } \\
\text { type }\end{array}$ & $\begin{array}{c}\text { No. trials } \\
\text { to criterion }\end{array}$ & $\%$ correct & Correct trials \\
\hline Whiskey & 670 & & \\
Training & & 83 & $20 / 24$ \\
Object transfer & & 92 & $22 / 24$ \\
Fabric transfer & & 92 & $22 / 24$ \\
Food transfer & & 75 & $18 / 24$ \\
Liza & 642 & & \\
Training & & 96 & $23 / 24$ \\
Object transfer & & 79 & $19 / 24$ \\
Fabric transfer & & 92 & $22 / 24$ \\
Food transfer & & 54 & $13 / 24$ \\
Opal & 951 & & \\
Training & & 75 & $18 / 24$ \\
Object transfer & & 83 & $20 / 24$ \\
Fabric transfer & & 75 & $18 / 24$ \\
Food transfer & & 62 & $15 / 24$ \\
Frieda & 1,002 & & \\
Training & & 88 & $21 / 24$ \\
Object transfer & & 88 & $21 / 24$ \\
Fabric transfer & & 79 & $19 / 24$ \\
Food transfer & & 79 & $19 / 24$ \\
Total & $M=816$ & & $83 / 96$ \\
Training & & 86.5 & $82 / 96$ \\
Object transfer & & 85.4 & $81 / 96$ \\
Fabric transfer & & 84.4 & $65 / 96$ \\
Food transfer & & 67.7 & \\
\hline Tote. Training sessions & & & \\
\hline
\end{tabular}

Note. Training sessions were the last two 12-trial sessions before transfer tests. Each transfer category included two 12-trial sessions, each session with two novel items.

The decline in performance in the case of food items was attributable to specific preferences, particularly in the case of 2 subjects, Liza and Opal, that had not appeared in the pretest preference testing reported earlier. During transfer tests, the probability of an animal's selecting the same food item regardless of its correctness was greater than $.84(p<.05$, binomial test). Had the animals simply failed to transfer, we would have expected the items to be chosen equally often as was the case during the paired-comparison forced-choice tests. Despite these preferences, both Whiskey and Frieda responded above chance ( $p<.05$, binomial test) with the food transfer items.

We also examined three aspects of the animals' performances on transfer tests that bear on the effects of differential feedback on breadth of construal. Specifically, these were (a) Trial 1 performance, when the animal first encountered a stimulus pair; (b) the effects of rewarding an incorrect Trial 1 choice on subsequent performance; and (c) evidence for a practice effect as reflected in performance differences between early and later trials in a session.

The overall correct MTS performance level on Trial 1 of each session with a novel stimulus pair was $77 \%(p<.05$, binomial test). Whiskey and Liza were correct on Trial 1 of all 6 transfer sessions. Opal made a correct Trial 1 matching response on one session each of the objects, fabric, and food transfer tests. Frieda was correct on Trial 1 in one session each of objects and food and on both sessions with fabrics.
For the five sessions beginning with an incorrect Trial 1 choice, the overall mean correct performance level was $79 \%$. This did not differ significantly from the correct performance level of $78 \%$ for those sessions beginning with a correct Trial 1 choice. The overall probability of correctly responding on the remaining 11 trials of a session after a correct Trial 1 choice was .77. After an incorrect Trial 1 choice, the overall correct response probability on the remaining 11 trials alone was .85 . This difference was not statistically significant.

There was no evidence of a practice effect on performance in the transfer tests. The animals' overall $81 \%$ correct MTS performance on trials in the first half (i.e., 132 trials) of each transfer session did not differ significantly from their overall correct performance level of $82 \%$ on trials in the second half of each transfer session.

The chimpanzees showed no improvement over the course of the 200 trials when the relational matching procedure was used. They responded at chance levels in both the initial 25 and final 25 trials. All 4 animals continued to respond at chance for the additional 50 trials with the relational MTS procedure in which the experimenter provided explicit overt cues indicating the correct matching choice. There was no relation between the animals' performances and the experience or familiarity of the trainers in either the relational MTS task or the transfer tests.

\section{Discussion}

All 4 chimpanzee infants acquired MTS after 642-1,002 training trials restricted to two metallic objects, a lock and a cup. These data are comparable to those reported previously in other studies using chimpanzee subjects (e.g., Finch, 1942; Nissen et al., 1948).

After acquisition of the MTS task with the two training objects, each animal immediately transferred her or his matching skills to novel items in the absence of explicit differential reward. Errors, when they did occur, were attributable to stimulus preferences. It is not immediately apparent why a preference should have developed during transfer testing with food items. However, an asymmetry in the response requirements of the preference testing situation and the MTS task may have played a role. During the initial preference testing, the chimpanzees readily relinquished the chosen objects or fabric swatches, whereas they were allowed to consume the selected object when food preferences were tested. During transfer testing of MTS, however, the animals had to give up a food item as they did also with objects and fabric comparison stimuli. The presumably greater motivational saliency of the food items may have led the animals to make a preference judgment during MTS transfer testing that was not evoked during preference testing.

The present transfer data are consistent with those reported by Nissen et al. (1948) and further demonstrate that like children and unlike old- and new-world monkeys, the chimpanzee's ability to match new items is not constrained when training is limited to a single object pair used interchangeably and repeatedly as matching and nonmatching alternatives. Having mastered the matching rule, the chimpanzee can apply it broadly to objects independently of their physical similarity 
to the original training items. For example, all subjects quite readily matched soft swatches of fabric after training with two metallic objects.

Immediate transfer of the matching concept is all the more impressive because we did not find an initial decrement in performance when transfer testing was begun. Temporary decrements resulting from perceptual exploratory learning about the new, if not novel, stimuli might be expected (D'Amato et al., 1985). If such perceptual learning about new test items is necessary, our data indicate that it can occur outside the matching task context. All of our subjects were preexposed to the transfer test items during preference testing in a freeplay situation.

There is no evidence that subtle cues from either familiar, experienced or unfamiliar, experimentally naive trainers signaled the correct response to the chimpanzees. If success on the transfer tests was independent of the stimulus objects but resulted instead from unintentional experimenter cuing, then we might reasonably have expected the chimpanzees to succeed also on the relational MTS task and their performance to vary with trainer. The animal's failure to match relations is consistent with past reports using nonlanguage-trained chimpanzees (Premack, 1983b). This result and their continued failure, even when explicit cues were provided by the trainer, makes it unlikely that such cues mediated the chimpanzees' successful matching in the transfer tests.

The present results convincingly demonstrate that experimenter-provided differential feedback was not necessary for transfer of the MTS response to new items. Even though incorrect Trial 1 choices in transfer tests were explicitly rewarded, this outcome did not determine further responding to that particular pair. Nor was there any evidence of a practice effect during transfer testing. Correct transfer performance levels did not vary within a session.

The present data provide strong evidence that the infant chimpanzees had acquired a broadly construed matching concept as suggested by Premack (1983a, 1983b). By this we mean a tendency to use a perceptual hypothesis instrumentally, independent of any particular exemplar. The chimpanzees' ability to match new object classes immediately is not what one would expect if they had learned initially a narrowly construed concept limited to the training items that then could be broadened only by experience. Their failure to transfer to the relational MTS task indicates that the concept is confined to physical resemblance and does not extend to relations. This latter finding, not altogether unexpected, supports Premack's (1983b) claim that the representational coding of identity between objects is not isomorphic with coding of identity between abstract relations.

Our results support Nissen et al.'s (1948), earlier suggestion that the critical factor in each MTS transfer trial is the "immediate perception of likeness and/or of difference" (p.71, emphasis supplied) within the situation. According to this viewpoint, rewards may function primarily to maintain arousal level and interest in performing the task per se; they do not necessarily determine individual transfer-trial choices. An alternative argument is that transfer of MTS to new items occurs through the association of increasing numbers of absolute stimulus dimensions to the matching response by re- ward (cf. Carter \& Werner, 1978; Schrier \& Thompson, 1980). Because nondifferential reinforcement was used in this study, the subjects' immediate transfer and response patterns within transfer sessions could not have been controlled in any simple associative way by reward.

Furthermore, the immediate transfer to items orthogonal to the training items indicates that reward was not functioning in any simple associative way during acquisition. During acquisition, the animal must attend to the sample and learn that her or his choice is predicated on the similarity between the sample and choice items. All other response hypotheses and perceptual invariances must be ignored. For example, reward leads the animal to reject the response alternative of picking the odd (i.e., nonmatching) item or any item that is consistently to the animal's left or right (cf. Harlow, 1949). However, the reward does not determine or instruct the chimpanzee as to whether this judgment should be based on the absolute stimulus values (e.g., see lock, pickup lock) or the more general basis of physical resemblance. This latter distinction is independent of the prevailing contingencies. Why these chimpanzee infants should focus spontaneously on physical resemblance rather than absolute stimulus values is not immediately clear from the present data; it may be a natural predisposition of the species (cf. Davenport \& Menzel, 1960). There was certainly nothing in these animals' experiences which would have biased them against reliance on absolute stimulus values.

As we suggested earlier, a critical comparative issue concerns the conditions necessary for a particular species to acquire and express a broadly construed concept. Our data suggest that in the case of the chimpanzee, rewards serve to confirm use of a general matching concept rather than simply to strengthen an approach/avoid response controlled by absolute stimulus values. Much of the available evidence cited earlier suggests that whatever capacity other organisms might have for using physical resemblance as a basis for MTS, they are predisposed to attend to absolute stimulus values. If their environment is structured in such a way that absolute stimulus values are not as salient-as, for example, when large numbers of stimuli are employed in MTS-some species will learn to match by using physical resemblance. Otherwise, in the absence of this form of environmental compulsion, the absolute cues most likely will control an animal's MTS performance. What is impressive about chimpanzees, as revealed in the present study, is that despite the opportunity to fall back on absolute cues, they are their own agents and spontaneously choose to use the more general principle of physical resemblance.

\section{References}

Carter, D. E., \& Werner, T. J. (1978). Complex learning and information processing by pigeons: A critical analysis. Journal of the Experimental Analysis of Behavior, 29, 565-601.

Daehler, M. W., Lonardo, R., \& Bukatko, D. (1979). Matching and equivalence judgements in very young children. Child Development, 50, 170-179.

D'Amato, M. R., \& Colombo, M. (1985). Auditory matching-tosample in monkeys (Cebus apella). Animal Learning \& Behavior, 13, 375-382.

D'Amato, M. R., \& Salmon, D. P. (1984). Cognitive processes in 
cebus monkeys. In H. L. Roitblat, T. G. Bever, \& H. S. Terrace (Eds.), Animal cognition (pp. 149-168). Hillsdale, NJ: Erlbaum.

D'Amato, M. R., Salmon, D. P., \& Colombo, M. (1985). Extent and limits of the matching concept in monkeys (Cebus apella), Journal of Experimental Psychology: Animal Behavior Processes, 11, 3551 .

D’Amato, M. R., Salmon, D. P., Loukas, E., \& Tomie, A. (1986). Processing of identity and conditional relations in monkeys (Cebus apella) and pigeons (Columba livia). Animal Learning \& Behavior, 14, 365-373.

Davenport, R. K., Jr., \& Menzel, E. W. (1960). Oddity preferences in the chimpanzee. Psychological Reports, 7, 523-526.

Edwards, C. A., Jagielo, J. A., \& Zentall, T. R. (1983). "Same/ different" symbol use by pigeons. Animal Learning $\&$ Behavior, 11, 349-355.

Farthing, G. W., \& Opuda, M. J. (1974). Transfer of matching-tosample in pigeons. Journal of the Experimental Analysis of Behavior, 21, 199-213.

Finch, G. (1942). Delayed matching-from-sample and nonspatial delayed response in chimpanzees. Journal of Comparative and Physiological Psychology, 34, 315-319.

Fodor, J. A. (1975). The language of thought. Cambridge, MA: Harvard University Press.

Fujita, K. (1982). An analysis of stimulus control in two-color matching-to-sample behaviors of Japanese monkeys (Macaca fuscata). Japanese Psychological Research, 24, 124-135.

Fujita, K. (1983). Formation of the sameness-difference concept by Japanese monkeys from a small number of color stimuli. Journal of the Experimental Analysis of Behavior, 40, 289-300.

Harlow, H. F. (1949). The formation of learning sets. Psychological Review, 56, 51-65.

Herman, L. M. \& Gordon, J. A. (1974). Auditory delayed matching in the bottlenose dolphin. Journal of the Experimental Analysis of Behavior, 21, 19-26.

Holmes, P. W. (1979). Transfer of matching performance in pigeons. Journal of the Experimental Analysis of Behavior, 31, 103-114.

Jackson, W. J., \& Pegram, G. V. (1970a). Acquisition, transfer, and retention of matching by rhesus monkeys. Psychological Reports, $27,839-846$.

Jackson, W. J., \& Pegram, G. V. (1970b). Comparison of intra- vs. extradimensional transfer of matching by rhesus monkeys. Psychonomic Science, 19, 162-163.

Johansson, G. (1964). Perception of motion and changing form. The Scandinavian Journal of Psychology, 5, 181-208.

Lashchyk, E. (1986). Heuristics for scientific and literary creativity: The role of models, analogies and metaphors. In J. Margolis, M. Krawsz, \& R. M. Burian (Eds.), Rationality, relativism and the human sciences (pp. 151-185). Dordrecht, The Netherlands: Martinus Nijhoff.

Mello, N. K. (1971). Alcohol effects on delayed matching to sample performance by rhesus monkeys. Physiology and Behavior, 7, 77101.

Meltzoff, A. N., \& Borton, R. W. (1979). Intermodal matching by human neonates. Nature, 282, $403-404$.

Milner, A. D. (1973). Matching within and between sense modalities in the monkey (Macaca mulatta), Joumal of Comparative and Physiological Psychology, 83, 278-284.

Mishkin, M., \& Delacour, J. (1975). An analysis of short-term visual memory in the monkey. Journal of Experimental Psychology: Animal Bchavior Processes, 1, 326-334.

Nissen, H. W., Blum, J. S., \& Blum, R. A. (1948). Analysis of matching behavior in chimpanzee. Journal of Comparative and Physiological Psychology, 41, 62-74.

Oden, D. L., Thompson, R. K. R., \& Premack, D. (1988). Perception of concrete and relational identity is immediate and direct in infant chimpanzees (Pan troglodyles). Manuscript submitted for publication.

Overman, W. H., \& Doty, R. W. (1980). Prolonged visual memory in macaques and man. Neuroscience, 5, 1825-1831.

Premack, D. (1978). On the abstractness of human concepts: Why it would be difficult to talk to a pigeon. In S. H. Hulse, H. Fowler, \& W. K. Honig (Eds.), Cognitive processes in animal behavior (pp. 423-451). Hillsdale, NJ: Erlbaum.

Premack, D. (1983a). Animal cognition. Annual Review of Psychology, 34, 351-362.

Premack, D. (1983b). The codes of man and beasts. The Behavioral \& Brain Sciences, 6, 125-137.

Premack, D., \& Woodruff, G. (1978). Does the chimpanzee have a theory of mind? The Behavioral \& Brain Sciences, 1, 515-526.

Ricciutti, H. (1965). Object grouping and selective ordering behavior in infants 12-24 months old. Merrill-Palmer Quarterly, 11, 129148.

Riesen, A. H., \& Nissen, H. W. (1942). Non-spatial delayed response by the matching technique. Journal of Comparative and Physiological Psychology, 34, 307-313.

Schrier, A. M., \& Thompson, C. R. (1980). Conditional discrimination learning: A critique and amplification. Journal of the Experimental Analysis of Behavior, 33, 291-297.

Shallice, T. (1972). Dual functions of consciousness. Psychological Review, 79, 383-393.

Spelke, E. S. (1979). Perceiving bimodally specified events in infancy. Developmental Psychology, 15, 626-636.

Steele, T. L., \& Bauer, R. H. (1983). A method for examining shortterm retention of haptic cues in monkeys. Behavior Research Methods \& Instrumentation, 15, 344-349.

Sternberg, R. J. (1977). Intelligence, information processing, and analogical reasoning. Hillsdale, $\mathrm{NJ}$ : Erlbaum.

Thompson, R. K. R. (1980). Auditory cued reversal and matchingto-sample learning by rhesus monkeys. Antropologia Contemporanea, 3, 284.

Wallach, H. (1958). On psychological similarity. Psychological Review, 65, 103-116.

Weinstein, B. (1941). Matching-from-sample by rhesus monkeys and by children. Journal of Comparative Psychology, 31, 195-213.

Wilson, B., Mackintosh, N. J., \& Boakes, R. A. (1985a). Matching and oddity learning in the pigeon: Transfer effects and the absence of relational learning. The Quarterly Journal of Experimental Psychology, 37B, 295-311.

Wilson, B., Mackintosh, N. J., \& Boakes, R. A. (1985b). Transfer of relational rules in matching and oddity learning by pigeons and corvids. The Quarterly Journal of Experimental Psychology, 37B, 313-332.

Worsham, R. W. (1975). Temporal discrimination factors in the delayed matching-to-sample task in monkeys. Animal Learning \& Behavior, 3, 93-97.

Zentall, T. R., Edwards, C. A., Moore, B. S., \& Hogan, D. E. (1981). Identity: The basis for both matching and oddity learning in pigeons. Joumal of Experimental Psychology: Animal Behavior Processes, 7, 70-86.

Zentall, T. R., \& Hogan, D. E. (1974). Abstract concept learning in the pigeon. Journal of Experimental Psychology, 102, 393-398.

Zentall, T. R., \& Hogan, D. E. (1978). Same/different concept learning in the pigeon: The effect of negative instances and prior adaptation to transfer stimuli. Journal of the Experimental Analysis of Behavior, 30, 177-186.

Received May 26, 1987

Revision received August 12, 1987

Accepted August 20, 1987 